\title{
Strates
}

STRATES Matériaux pour la recherche en sciences sociales

$10 \mid 2001$

Villageois et citadins de Grèce

\section{Les conflits pour l'eau en Étolie-Acarnanie}

Chocs entre État et initiative individuelle, émergence d'une identité régionale

Michel Bouillet

\section{CpenEdition}

1 Journals

Édition électronique

URL : http://journals.openedition.org/strates/367

DOI : $10.4000 /$ strates.367

ISSN : $1777-5442$

Éditeur

Laboratoire Ladyss

Édition imprimée

Date de publication : 1 mai 2001

ISSN : 0768-8067

Référence électronique

Michel Bouillet, «Les conflits pour l'eau en Étolie-Acarnanie », Strates [En ligne], 10 | 2001, mis en ligne le 11 janvier 2005, consulté le 07 septembre 2020. URL : http://journals.openedition.org/strates/367 ; DOI : https://doi.org/10.4000/strates.367

Ce document a été généré automatiquement le 7 septembre 2020

Tous droits réservés 


\section{Les conflits pour l'eau en Étolie- Acarnanie}

Chocs entre État et initiative individuelle, émergence d'une identité régionale

\section{Michel Bouillet}

Un potentiel hydraulique abondant

1 Le nome (département) d'Étolie-Acarnanie, 228180 habitants au recensement de 1991, est situé sur la façade occidentale de la Grèce. Il présente un caractère relativement peu courant dans le monde méditerranéen par l'abondance de ses ressources hydriques naturelles. En effet, sur ses côtes son climat est tempéré par l'influence adoucissante de la mer Ionienne, tandis que la continentalité affecte les montagnes et les bassins intérieurs (plaine d'Agrínio, vallée du Bizákos). Les températures sont caractérisées par des amplitudes notables : $11^{\circ} 1$ pour la moyenne du mois le plus froid et $23^{\circ} 3$ pour le plus chaud à Agrínio. Toutefois, en raison des variations inter- annuelles très importantes, les minima et les maxima absolus y sont de $-7^{\circ} 3$ et de $+44^{\circ} 8$. Cette ville par temps de canicule est une véritable fournaise, phénomène habituel en Grèce continentale. 


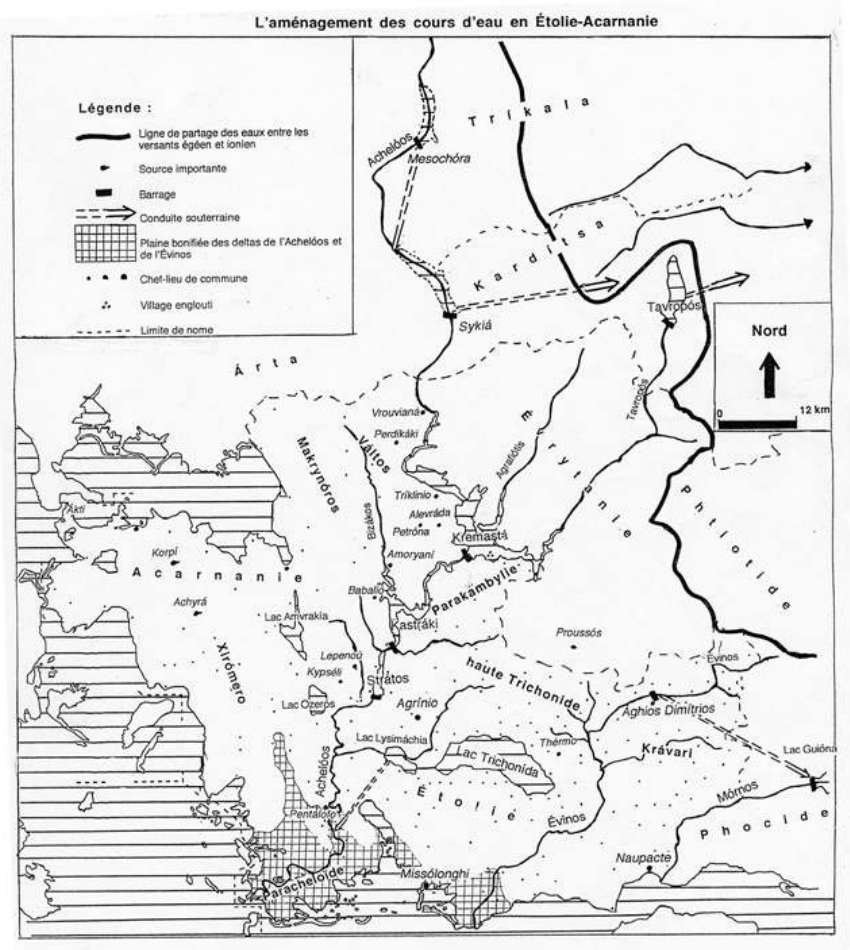

2 Les dépressions hivernales sont gages de précipitations abondantes sur la façade maritime. Bien orientées face aux flux de sud-ouest, les stations d'Ákti et de Naupacte reçoivent en moyenne respectivement 930 et $944 \mathrm{~mm}$ d'eau par an alors que Missolónghi ne bénéficie que de $737 \mathrm{~mm}$ et qu'Agrínio monte à $1027 \mathrm{~mm}$, profitant du microclimat plus humide engendré par la proximité du lac Trichonída. Ces valeurs sont supérieures à celles relevées sur le versant égéen (340 mm à Athènes, 416 à Salonique) et dans le Péloponnèse ( $616 \mathrm{~mm}$ à Pátras). Les précipitations se répartissent sur l'année selon un schéma HAPE. Á Ákti, 80 \% de la quantité d'eau annuelle tombe entre octobre et avril. Néanmoins, les précipitations sont encore à craindre en mai et les orages d'été peuvent être redoutables, en dépit d'une aridité dominante en saison chaude ${ }^{1}$. Les chutes de neige sont rares et de faible abondance en plaine, mais habituelles et beaucoup plus conséquentes en altitude: la route de Thérmo à Proussós est fréquemment coupée en hiver.

3 Les montagnes ont donné naissance à des cours d'eau torrentiels d'orientation subméridienne, à savoir d'ouest en est, l'Achelóos (220 km de long, deuxième fleuve hellénique, artère principale du nome et limite historique entre l'Étolie et l'Acarnanie), l'Évinos $(40 \mathrm{~km})$ et le Mórnos (70 km). S'ajoute le plus vaste ensemble lacustre du pays, avec une superficie supérieure à $145 \mathrm{~km}^{2}$ ( $24 \%$ du total grec), dont les lacs Trichonída (le plus étendu du pays avec $95,84 \mathrm{~km}^{2}$, profondeur maxima: $57 \mathrm{~m}$ ), Amvrakía, Lysimáchia et Ozerós, complété par trois lacs artificiels sur l'Achelóos: Kremastá, Kastráki et Strátos (près de $51 \mathrm{~km}^{2}$ ). L'Étolie-Acarnanie peut prétendre au titre de " château d'eau de la Grèce ». Pour l'ensemble de la Grèce occidentale, l'écoulement superficiel se monte à $20,2 \mathrm{~km}^{3}$, soit près du tiers de l'ensemble de l'Hellade ${ }^{2}$, sans compter les réserves souterraines évaluées à $0,13 \mathrm{~km}^{3}$.

4 Ce potentiel hydraulique exceptionnel a été exploité traditionnellement selon des techniques rudimentaires étonnantes en milieu méditerranéen, ailleurs si propice à l'ingéniosité dans le développement de techniques complexes d'irrigation. 
Des techniques individuelles, rudimentaires et ingénieuses

Ces techniques ont été développées à l'initiative du paysan, comme le notait déjà le perspicace Edmond About en 1854 : « Les paysans grecs sont très habiles à tirer parti du moindre ruisseau pour arroser leurs plantations ${ }^{3}$.» Déjà à l'époque, les moindres sources dans les séries de flysch ${ }^{4}$ étaient captées et dirigées vers les minuscules champs, installés sur des replats naturels ou des terrasses. Aujourd'hui, les tuyaux en plastique ou les canalisations métalliques à raccords rapides serpentent le long des chemins rocailleux du Makrynóros ou de la haute Trichonide et les anciennes rigoles sont parfois encore utilisées, même si elles sont rarement empierrées. Ici, au sud du Pinde et dans son piémont, l'eau sourd en de nombreux points, jamais en grande quantité (on est loin des exurgences des pays calcaires), mais finalement de manière abondante et pérenne. Le sous-peuplement du Váltos et de la haute Trichonide ${ }^{5}$ peut expliquer le caractère rudimentaire de cette forme d'irrigation. Une technique plus élaborée, à l'image du dir d'Afrique du Nord ou des parats catalans, comportant des rigoles maçonnées ${ }^{6}$, aurait nécessité une organisation sociale plus collective et une main-d'œuvre plus nombreuse. L'ensemble de la communauté clanique se déplace encore au gré des pâtures, vivant dans des kalyves, cabanes temporaires faites de roseaux ou de bois. C'est bien un nomadisme dans un cadre familial - et non une transhumance - qui a persisté tardivement dans le Makrynóros. Il était hors de question de se livrer à un travail collectif de longue haleine tel que celui nécessaire à l'élaboration de systèmes d'irrigation complexes. Dans le Krávari, montagne-refuge plus densément peuplée, l'étroitesse des terroirs et l'individualisme ont constitué un frein à l'entreprise de grands travaux au sein de montagnes-refuges, où, du temps de la turcocratie, un raid des Turcs ou des Albanais n'était pas à exclure.

6 Dans les bassins intérieurs du Xirómero calcaire, Heuzey (1860) décrit les loútses, dolines ou vastes trous d'eau creusés à main d'hommes, remplies par les pluies d'automne et d'hiver et utilisées l'été à abreuver les troupeaux ou à arroser les champs. Cet apport est indispensable au printemps lors du repiquage des jeunes plants de tabac. La terra rossa assure leur imperméabilité, complétée souvent par des feuilles de plastique agricole. Ces installations modernisées sont toujours en usage avec des pompes mobiles installées aux prises de force des tracteurs qui assurent le transport de l'eau à travers des tuyaux métalliques à raccords rapides vers les champs.

7 Un système agro-sylvo-pastoral montagnard à la recherche d'un équilibre, par définition instable, s'est instauré au fil des siècles dans le cadre de la communauté villageoise sur la base de la liberté et de la solidarité familiales. Le but est de récolter sur sa propriété, grâce à l'eau don du ciel, d'abord de quoi nourrir la famille, ensuite seulement des produits échangeables. Le monde extérieur proche était considéré comme a priori hostile: monde des plaines occupées par les Turcs jusqu'à l'indépendance, puis par les grands propriétaires, peuplé de rayas ${ }^{7}$ méprisés; monde d'où venaient les collecteurs d'impôts et l'autorité étatique; enfin, monde paludéen, malsain, à l'air vicié générateur de toutes les maladies.

8 Le peuplement progressif des plaines s'effectue sous l'impulsion d'une violente poussée démographique qui chasse des montagnes nombre d'hommes contraints au travail d'un lopin en plaine loin du village, ou encore dans le bas terroir de la commune si celle-ci en dispose ${ }^{8}$. L'eau est ici une ressource abondante à partir des sources captées et dirigées grâce à un réseau de petits canaux maçonnés, remarqués déjà par Bazin (1864) sur la rive méridionale du lac Trichonída. Elle provient également de la bonification 
aux $\mathrm{XIX}^{\mathrm{e}}$ et $\mathrm{XX}^{\mathrm{e}}$ siècles des vastes marécages occupant la plaine centrale étolienne, aujourd'hui traversée par un réseau de canaux et vouée au tabac et au maïs. Enfin, les lacs sont abondamment utilisés par des stations de pompage individuelles et parfois même, comme c'est le cas au bord du lac Amvrakía, les cultures en occupent à la belle saison les rives laissées libres par le retrait des eaux. Il est à souligner que ce travail est le fruit essentiellement des efforts individuels ou de communautés villageoises. Ainsi, la vision du monde engendrée par le système agro-sylvo-pastoral s'est-elle maintenue en plaine dans un cadre spécifiquement agricole et spéculatif, amplifiée par l'émigration lointaine vers les villes étolo-acarnaniennes, Athènes ou l'étranger. Le cœur en est constitué par la maîtrise de l'apport hydrique indispensable pour passer l'été et pour nourrir les jeunes plants de tabac, spéculation primordiale du nome, ainsi que par la prééminence de l'effort individuel reposant sur une conception viscérale de la liberté et une méfiance séculaire à l'égard de l'État.

Les grands travaux d'aménagement hydraulique : satisfaire des besoins nationauxL'aménagement de l'Achelóos de 1918 à 1989 : le choc de la modernité

Les tout premiers grands travaux intéressent l'Achelóos, artère centrale du nome d'Étolie-Acarnanie. L'objet des concepteurs n'était pas tant d'irriguer, contrairement aux aspirations des agriculteurs locaux, que d'assurer la régularité de la production hydroélectrique ${ }^{9}$, en maitrisant les très fortes variations saisonnières et inter-annuelles du débit d'un cours d'eau méditerranéen.

10 Les premiers projets d'aménagement hydroélectrique du fleuve datent des années 1918 à 1921, mais c'est seulement en novembre 1961 que le premier coup de pioche fut donné, pour un chantier achevé en $1966^{10}$. Le coût total du barrage et de l'usine hydroélectrique de Kremastá s'est élevé à 81770000 dollars. Somme considérable, qui, pour être remboursée, nécessite d'exporter du courant. Les départements montagnards de Grèce occidentale sont incapables d'absorber une production prévue de $1430 \mathrm{GWh}$. L'éloignement des centres de consommation a imposé l'édification de lignes à haute tension, vers le nord et l'ouest. Les impératifs de rentabilité financière ne passaient pas par l'Étolie-Acarnanie, espace transparent de ce point de vue.

11 Kremastá a été construit à l'entrée des gorges du même nom, solidement appuyé sur des assises calcaires. D'une hauteur maxima de $160,3 \mathrm{~m}$ et d'une longueur de $287 \mathrm{~m}$, il développe un lac artificiel de $80 \mathrm{~km}^{2}$, contenant un volume de 4500 millions de $\mathrm{m}^{3}$ d'eau, remontant en trois branches vers l'amont de l'Achelóos, de l'Agrafiótis et du Tavropós. C'est le deuxième lac du nome derrière Trichonída.

12 L'aménagement de l'Achelóos a été poursuivi, de 1965 à 1969, par la construction, à l'aval de Kremastá, du barrage de Kastráki, de dimensions plus modestes. Construit en terre, tandis que le précédent est un barrage-voûte en béton armé, il développe une hauteur de $95 \mathrm{~m}$. Il a ennoyé le moyen Achelóos et le bas Bizákos, formant un lac de $28 \mathrm{~km}^{2}$, d'une capacité de 900 millions de $\mathrm{m}^{3}$.

13 Enfin, $8 \mathrm{~km}$ au sud de Kastráki, l'ouvrage de Strátos a été commencé en 1981 par une entreprise grecque de terrassement, qui a dû abandonner le chantier au bout de deux ans et demi, suite à des problèmes de trésorerie. Il a été achevé par l'entreprise italienne Torno, et les deux tranches que comporte l'installation électrique ont débuté la production en 1988 et 1989. Haut de $26 \mathrm{~m}$, son lac réservoir contient 80 millions de $\mathrm{m}^{3}$ et s'étend sur $7,4 \mathrm{~km}^{2}$. $10 \%$ du potentiel national. Les trois barrages édifiés sur l'Achelóos en constituent la 
quasi-totalité : Kremastá, en amont, possède l'usine hydroélectrique la plus importante avec 437 MW, Kastráki figure au deuxième rang avec 320 MW et enfin Strátos, en aval, aligne $156 \mathrm{MW}$. L'ensemble atteint $37 \%$ de la puissance hydroélectrique installée en Grèce, faisant du département le premier du pays et le second pour la fourniture de courant électrique, toutes origines confondues, si l'on compte les installations thermiques, loin derrière Kozáni, animé par le lignite de Ptolemaïda (4 $481 \mathrm{MW}$ ).

La production, pour importante qu'elle soit, n'atteint pas les prévisions du fait de l'insuffisante alimentation hydrique des turbines. Ainsi, le niveau de l'eau dans le réservoir de Kremastá se situait, en 1993, à 20 mètres en-dessous de la normale. De même, la capacité annuelle annoncée pour Strátos de $400 \mathrm{GWh}$, n'a pas été approchée : en 1992 les deux unités, Strátos I et II, ont fourni 183 GWh.

Il faut souligner que l'aménagement de l'Achelóos est à l'origine de mouvements de population significatifs affectant les communes bordières entre 1960 et 1989. La commune d'Ághios Vassílios disparaît, alors que cinq autres perdent leurs meilleures terres cultivables avec l'édification de Kremastá11. La population est transférée principalement dans la plaine d'Agrínio. Comme dans les années 1923 à 1928 avec l'installation des réfugiés d'Asie Mineure et du Pont, le barrage, en liaison avec le risque sismique, sert de justification à une politique autoritaire d'aménagement du territoire pour l'ensemble des communes du haut Váltos et de Parakambylie ${ }^{12}$. Après la mise en eau de Kremastá, et pendant la dictature des colonels, les déplacements se multiplient ${ }^{13}$. Ils se poursuivront jusque dans les années quatre-vingt et toucheront au total 2077 familles ayant obtenu des prêts ou des aides à la mobilité et à la réinstallation ${ }^{14}$. D'autres villages sont complètement destructurés. Ainsi, le barrage de Kastráki ennoie Babalió et une partie d'Amoryianí. Babalió perd la moitié de sa population entre 1961 et 1971, le reste étant regroupé au chef-lieu. Amoryianí est restructurée autour du nouveau village, qui voit sa population augmenter de $8 \%$ dans le même temps, pendant que ses hameaux de Malatéiko et Prantikó s'effondrent, perdant $80 \%$ de leurs habitants.

Le bilan démographique fait ressortir la déperdition accélérée des communes dont une partie de la population a été dirigée hors de leurs limites administratives : elles ont perdu en moyenne $56 \%$ de leur population entre 1961 et 1991. Á l'inverse, les villages déplacés à l'intérieur du finage communal ont ralenti significativement leur déclin : la diminution a été limitée à $11,5 \%$ durant la même période ${ }^{15}$. En outre, parmi les communes du Váltos exclusivement situées en altitude - entre 500 et 740 mètres Alevráda, Petróna et Tríklinio déclinent plus rapidement pendant la dernière période intercensitaire - et elles sont aujourd'hui moribondes - que Vrouvianá et Perdikáki qui n'ont pas été l'objet d'une planification des départs. Un tel bouleversement humain induit par les grands travaux étatiques a été considéré comme le prix à payer pour l'industrialisation du pays. Certes, une partie des populations déplacées a pu trouver des terres irrigables et mieux valorisables, mais le reste (la majorité ?) était définitivement déracinée.

18 L'irrigation à partir des lacs-réservoirs est strictement réglementée. La prise d'eau individuelle est interdite, et la loi est respectée. L'eau est vendue à bon marché par les GOEV $^{16}$ aux exploitants, pour couvrir les frais d'entretien des canaux ${ }^{17}$. Les agriculteurs de la plaine d'Agrínio et du couloir étolien bénéficient d'apport d'eau évalué à 250 millions de $\mathrm{m}^{3}$. Cependant celui-ci ne va pas sans difficulté. Les relations entre les différents partenaires se sont dégradées sur fond de concurrence pour une ressource 
raréfiée par des années successives de moindre pluviométrie et par une demande croissante provoquée par les besoins antagonistes de cultures de plus en plus massivement irriguées et d'une régulation des cours du fleuve de plus en plus difficile à obtenir pour assurer le bon fonctionnement des turbines, alors que la demande de courant électrique explose. Il s'ensuit des désillusions de la part des agriculteurs se manifestant par des refus de payer l'eau et la cotisation au TOEV, ainsi que par des actions en justice intentées par les syndicats locaux à la $\mathrm{DEI}^{18}$ afin de l'obliger à restaurer les canaux passablement dégradés faute d'un entretien régulier, comme à Lepenoú en 1997.

19 Ainsi, le passage d'un système d'apport d'eau «traditionnel» à un système "moderne », imposant une cogestion entre utilisateurs privés et société publique par l'intermédiaire d'organismes paritaires ne s'effectue pas correctement. À l'origine des conflits se posent les questions complexes de la charge de chacun dans l'entretien des canaux. La dilution des responsabilités entre les partenaires à un moment où le monde agricole subit une mutation profonde et une baisse conséquente de ses revenus liée à la baisse des prix et au désengagement de l'État imposé par la volonté affichée d'intégration à l'Union européenne, dilution favorisée par des gestions souvent laxistes de la part des organismes étatiques, a provoqué des situations inextricables se manifestant par les refus de paiement ou les actions en justice, mais également par des levées de fourches périodiques, comme celles de l'hiver 1996. Au quotidien, la réaction des exploitants agricoles consiste au repli sur soi en forant son propre puits sans en référer à quiconque, retrouvant instinctivement les réflexes ancestraux.

Des grands travaux contemporains inadaptés aux nécessités locales

20 Depuis l'achèvement des barrages sur le territoire étolo-acarnanien, le cours de l'Achelóos a été l'objet de nouveaux travaux sur son cours supérieur dans les départements de Tríkala et de Kardítsa. Deux nouveaux barrages sont en construction à Mesochóra et à Sykiá, afin d'alimenter deux nouvelles usines hydroélectriques. Un cinquième à un tiers des eaux de l'Achelóos doit être détourné par un tunnel de dérivation vers la plaine thessalienne, afin de satisfaire les besoins hydriques des planteurs de coton et des citadins. Ces ouvrages ont été partiellement financés par la CEE dans le cadre du PIM $^{19}$ Grèce Centrale, et non dans celui concernant la Grèce occidentale, lequel avait prévu des travaux de bonification dans la plaine du Lessíni, c'est-à-dire du bas Achelóos. La contradiction entre ces deux catégories de projets, demandeurs de la même eau du fleuve, laisse songeur quant à la cohérence de la vue d'ensemble.

21 Le deuxième grand ouvrage affectant l'Étolie-Acarnanie barre le cours amont de l'Évinos à Ághios Dimítrios et permet le détournement des eaux vers le Mórnos par un tunnel de dérivation sous le Tsekoúra. Le collectage des eaux doit s'effectuer dans l'actuel lac-réservoir de Guióna en Phocide. De là, elles sont acheminées depuis 1985 par un aqueduc vers Athènes.

Les études sur le terrain ont débuté en 1991, les travaux l'année suivante et sont en voie d'achèvement en 1997. Le consortium international assurant les travaux emploie pour partie des Étolo-Acarnaniens, mais également des Colombiens, embauchés pour la circonstance. Après cinq années de travaux ayant transformé la route qui monte du delta du Mórnos, où se trouvent les stations de concassage alimentant les stations à béton à Ághios Dimítrios, en un enfer de poussières et de trafic, le réseau routier s'est nettement amélioré. Les pistes cèdent la place à des voies goudronnées. 

avant son allongement. Elle doit permettre d'éviter les pertes, de satisfaire le consommateur été comme hiver, d'améliorer le goût et la qualité bactériologique de l'eau. Cependant, ces travaux sont coûteux. Il est nécessaire de mobiliser des crédits sans que le retour sur investissements ne soit assuré à brève échéance. C'est ce lieu commun de tout progrès technique généralisé à l'ensemble de la population, qui définit la problématique actuelle des infrastructures étolo-acarnaniennes. Au nom de l'égalité de tous devant la disponibilité en eau et de l'économie d'une ressource aussi précieuse, il faut répartir les charges le plus largement possible, donc augmenter les tarifs, et planifier les travaux de la manière la plus rationnelle possible. Les autorités locales sont placées désormais devant l'obligation de prendre des mesures impopulaires.

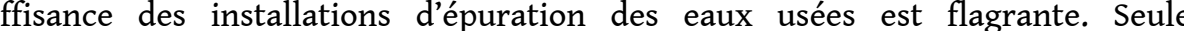
Missolónghi s'est dotée d'un système d'évacuation entre 1972 et 1975, car il était inclus dans un vaste plan de bonification des terres de la commune et de Paracheloïde, ce qui était beaucoup plus important pour assurer une clientèle reconnaissante aux autorités de l'époque. Les travaux avaient été confiés à l'entreprise EDOK-ETER AE, qui a réalisé la dérivation du lac Lysimáchia vers la Paracheloïde. Mais la cité ne dispose toujours pas d'une station moderne de retraitement et d'élimination des déchets. La pollution du golfe d'Ambracie et des lacs où arrivent les rejets urbains, notamment ceux d'Agrínio déversés dans le lac Lysimáchia, n'est pas évaluée de manière suffisamment systématique ni transparente. Les données publiées sont d'une extrême minceur ${ }^{22}$. Néanmoins, ce problème existe. Il est évoqué très succinctement dans des rapports d'études ${ }^{23}$ et plus précisément par nombre d'interlocuteurs sur le terrain. En outre, on peut le remarquer par de simples observations de bon sens. Néanmoins, la commune d'Agrínio avait en projet en 1993 la construction d'une station d'épuration.

Le rejet de polluants d'origine agricole est implicitement reconnu à l'occasion de problèmes rencontrés à l'exportation par l'Organisme National du Tabac, par exemple, qui met en évidence l'abus d'utilisation des produits phytosanitaires, objets d'une 
propagande aiguë auprès des agriculteurs. Si leur présence anormale a été déterminée, où va le surplus non absorbé par les plantes? La prise de conscience écologique est patente parmi une fraction de la population. Les premiers ont été les jeunes et certains responsables locaux sensibles au rôle que pourrait jouer le tourisme dans l'économie locale. Ensuite les agriculteurs (comme, par exemple, ceux de la commune de Neochóri en Paracheloïde qui manifestent leur colère devant la mauvaise qualité de l'eau en refusant de régler la facture présentée par la TOEV et comme à Lepenoú) effectuent leurs propres forages sauvages. Cependant, ils sont sensibilisés à la qualité de l'eau essentiellement en raison de ses incidences économiques. Ils jugent leur production invariablement propre et saine. Ce thème, jusqu'à présent électoralement peu porteur, n'a pas encore engendré de mesures notables de la part des autorités, ne serait-ce que pour prendre conscience de l'étendue du problème.

La montée récente du sentiment régionaliste

Il est incontestable que l'Étolie-Acarnanie a beaucoup plus donné que reçu dans la course à la modernité menée autoritairement par les gouvernements grecs.

On vient de voir l'influence décisive des grands travaux sur l'exode rural, avec la déstructuration des terroirs communaux partiellement noyés par les eaux des réservoirs. Si certains ont rebâti leurs activités autour des villages reconstruits, comme à Amoryianí, d'autres ont réalisé les indemnités attribuées par l'État, correspondant, en moyenne, à une maison et dix strémmata, pour acheter un appartement à Agrínio ou à Athènes. Soit ils y ont installé leur nouvelle activité, soit ils en ont tiré un loyer. L'effet des aménagements hydrauliques ne correspond pas à ce qui avait été prévu par leurs concepteurs. Il n'est pas non plus entièrement négatif, comme tenterait de la laisser penser tout un courant écologiste, mais il doit être compris comme l'irruption de la modernité parmi des communautés rurales non préparées. La vallée de l'Achelóos se définit comme le "couloir de la modernité » perceptible, pas tant dans les chiffres d'évolution de la population, que dans les paysages où les terroirs semblent mieux exploités qu'ailleurs et laisser plus de place à de nouvelles spéculations. Les agriculteurs y manifestent une réactivité dynamique aux changements. Déjà, les descendants de réfugiés installés à Kypséli ont-ils réussi à avoir le quasi-monopole de la culture des fraises dans le nome. Plus récemment, des exploitants de Lepenoú se sont regroupés, hors des cadres coopératifs traditionnels, par affinités familiales et amicales, en une véritable «paréa productive ${ }^{24}$ » pour réorienter une partie de leur production vers la culture des asperges destinées à l'exportation vers Munich par l'intermédiaire d'une connaissance installée là-bas.

31 Les réactions de la population rurale peuvent se répartir dans un premier temps entre des comportements négatifs et revendicatifs d'une part (procès ${ }^{25}$, refus de paiement de l'eau, dénigrement des TOEV, manifestations violentes et spontanées à l'occasion d'un mouvement national à propos de problèmes généraux du monde agricole) et des comportements positifs (recherches personnelles de méthodes d'irrigation moins gourmandes, forages individuels, réorientation des activités productives). Il convient de remarquer - et cela rejoint les remarques de M. Sivignon à propos des producteurs de coton de Pharsale $^{26}$ - que les pouvoirs publics sont étrangers à ces réactions positives. Il est à noter également que les considérations de protection de l'environnement ne sont pas mises en avant et c'est incidemment que certaines mesures, comme le recours au goutte-à-goutte, sont adoptées, tandis que d'autres, dangereuses à terme, comme les forages individuels, se développent dans un même 
temps. D'ailleurs des cas de salinisation des terres à cause de ce procédé sans contrôle ont été signalés en Paracheloïde.

Néanmoins, les mentalités ont évolué depuis la construction du barrage de Kremastá. Quelles furent les réactions des populations à l'époque ? Une réponse précise est bien difficile à fournir. Toutefois, d'après les témoignages recueillis sur le terrain, il semble bien que la résignation fut de mise dans les années soixante et soixante-dix, à part quelques résistances individuelles présentées par des tenants de «l'archaïsme ». Cela paraît vraisemblable, tant la pression idéologique était puissante sur des populations accoutumées à subir les décisions de pouvoirs économiques et politiques lointains.

Dans les années quatre-vingt, la construction de Strátos a donné lieu à des manifestations. La destruction de bonnes terres, portant des cultures rémunératrices telles que le tabac, le maïs ou les fruits et légumes, est devenue un thème plus mobilisateur que la perte de maigres champs en polycultures. Mais un thème nouveau est apparu au grand jour à cette occasion, avec l'intervention d'écologistes allemands : la dégradation irrémédiable de biotopes naturels, tels que le delta de l'Achelóos, lieu d'hivernage de nombreuses espèces d'oiseaux migrateurs, ou les rives marécageuses du fleuve. Cependant, le barrage a été construit.

Dans les années quatre-vingt-dix, l'hostilité de la population se manifeste par des inscriptions vengeresses sur les murs, telle celle relevée à Ághios Dimítrios : «L'Évinos est parti, il est temps pour vous de partir. » Elle participe de deux registres : l'angoisse d'une pénurie du précieux liquide obérant les efforts des agriculteurs, et la destruction d'un patrimoine écologique d'autant plus précieux qu'il intéresse des étrangers. Cette conception patrimoniale du milieu s'inscrit dans une quête des racines perceptible dans les comportements et les paysages. Par exemple, pour ce jeune commerçant installé dans son village d'origine du Váltos après avoir poursuivi des études et avoir vécu quelques années à Athènes, et qui explique son retour au pays par le rapprochement avec un mode de vie moins trépidant et un cadre de vie moins pollué, même si cela doit lui coûter une perte de revenus. On peut le remarquer encore à travers l'effort architectural consenti par les bâtisseurs de maisons individuelles ou par les restaurateurs de chapelles ruinées, tous respectueux d'un style traditionnel et tournant le dos aux mornes cubes de béton qui firent florès pendant des décennies. La question de l'eau est en outre accentuée par une succession d'années moins pluvieuses et par des pompages croissants - pratiquement jamais remis en cause par les exploitants euxmêmes.

La réalisation dans ce contexte des grands travaux de détournement de l'Évinos vers le Mórnos et d'une partie du haut Achelóos vers la plaine thessalienne, auxquels est venu s'ajouter le projet grandiose d'aqueduc qui doit apporter l'eau du lac Trichonída vers Athènes ${ }^{27}$, a pour effet de provoquer une situation explosive en ajoutant une dimension politico-identitaire. Le rejet de ces grands chantiers est massif - à la ville comme à la campagne - comme si désormais la subordination des intérêts étolo-acarnaniens à l'intérêt général et la soumission aux oukases des pouvoirs extérieurs étaient des états de fait révolus. On s'oppose à la dispersion du patrimoine hydrique considéré comme appartenant aux riverains également au nom d'une identité étolo-acarnanienne. Cette identité a longtemps été perçue de manière très floue pour une région ni épirote, ni moraïte, mais confondue dans un vaste espace montagnard rouméliote. Aujourd'hui cette population n'est plus majoritairement montagnarde et rurale, mais elle a participé du mouvement de migration vers les plaines et elle s'est urbanisée. Si on peut 
s'interroger sur la part des lobbies agricoles thessaliens dans le processus décisionnel du déversement des eaux de l'Achelóos ${ }^{28}$, l'opposition est générale à la livraison de ce patrimoine aux habitants du versant égéen. Le sentiment d'appartenir à une région oubliée, périphérique, se trouve résumé par une banderole placée par les autorités communales du village de Pentálofo (rive droite du bas Achelóos) en 1993. On peut y lire: «Non au détournement de l'Achelóos. Non au marasme de l'Étolie-Acarnanie.» Les habitants rencontrés adhéraient pleinement à ce slogan mêlant la question de l'eau avec les difficultés économiques, lesquelles sont évidemment plus complexes.

L'Étolie-Acarnanie existe, non seulement en tant qu'entité administrative, mais désormais comme unité humaine cohérente sur fond de conflit entre les intérêts du versant ionien de la Grèce par rapport au versant égéen, et pas seulement d'Athènes. La méfiance envers Athènes qui "bouffe la Grèce » m'a été maintes fois exprimée en ces termes par de nombreux interlocuteurs, mais au sentiment traditionnel de jalousie et de mépris du Rouméliote pour le Péloponnésien - que transcrit le proverbe « Toutes les pierres que Dieu avait, il les a toutes jetées en Roumélie, et la terre il l'a gardée pour la Morée $^{29} ! »-$ s'ajoute le ressentiment à l'encontre des Thessaliens censés avoir été favorisés. Á la question juridique posée par le droit des populations riveraines à garder le bénéfice de leurs ressources naturelles, se superpose l'affirmation d'une identité, dont le préalable suppose toujours la différenciation par rapport à ses voisins. L'étude d'un tel phénomène ne peut guère reposer sur des données quantifiables en l'absence d'étude sociologique approfondie, c'est néanmoins une réalité perceptible par le géographe de terrain. Il permet d'évoquer à bon droit la naissance d'un régionalisme étolo-acarnanien.

\section{BIBLIOGRAPHIE}

About E. (1907), La Grèce contemporaine, $12^{\mathrm{e}}$ édition, Paris, 408 p. (1 $1^{\mathrm{re}}$ éd. 1854).

Arvanítis K. et Gatzélia A. (1990), « S¥sthma Mønimhq Parakoloyu $\Delta$ shq thq Poiøthtaq tvn Epifaneiak $\sqrt{n}$ Ydat $\sqrt{ } n$ - Protåsh Am ${ }^{\mathrm{Tm}}$ shq Efarmøghq », in Eku ${ }^{\mathrm{Tm}}$ seiq 1, Prøgramma Ambrakiko¥ (tome 4), Athènes, 468 p., p. 297-327.

Bazin M. (1864), « Mémoire sur l'Étolie », in Archives des Missions scientifiques et littéraires, tome I, p. 249-370.

Bernabé y Maestre J. M. (1989), « Obras hidraulicas tradicionales en el regadío de Petrer (Valle del Vinalopó) », in Los Paisajes del agua, libro jubilar dedicado al Pr. A. López, Universidad de Valencia.

Bouillet M. (1997), Des Montagnes du Pinde à la Plaine de l'Achelóos : hommes et activités en ÉtolieAcarnanie (Grèce), thèse, Université de Provence, Aix-en-Provence, 1997.

Davy L. (1990), «L'eau, atout ou limite au développement », in La CEE méditerranéenne, Collection Dossiers des images économiques du monde SEDES, Paris, p. 93-139. 
Drain M. (dir.) (1996), « Les conflits pour l'eau en Europe méditerranéenne », Espace rural, n 36, janvier, $263 \mathrm{p}$.

Heuzey L. (1860), Le Mont Olympe et l'Acarnanie, Firmin Didot, Paris 1860, p. 223-280.

Karaghéorgou E. D. (1966), Étude sur la sécurité des villages de Chalkiópouli Tríklinio, Alevráda et Petróna, Athènes (texte grec, inédit).

Loukópoulos D. (1983), Gevrgikå Thq Ro¥melhq, Éd. Dodóni, Athènes-Iánnina, 495 pages (1 $1^{\text {re }}$ éd. 1938).

Varia (1988), université de l'Égée, «Aitvloakarnaniåq, Parøn kai $\mathrm{M}^{\mathrm{TM}}$ Ilon », Préfecture d'Étolie-Acarnanie, Missolónghi.

Varia (1988), Nomarxºa Aitvloaq kai Akarnanªq, A Anaptyjiako¥ Synedrio¥ nomo¥ Aitvloakarnanªq, Mesoløggi 23-24 Septembrooy 1988, 252 p.

\section{NOTES}

1. Les variations inter-annuelles sont importantes également. La sécheresse de 1989 a été durement ressentie, d'autant que 1990 est resté en deçà de la moyenne décennale, et que l'année a été caractérisée par un effondrement des pluies en janvier, traditionnellement le mois le plus arrosé. Le manque d'eau est le premier sujet d'inquiétude durant les années 1990-1993, particulièrement en Acarnanie, remettant en cause les efforts de développement fondés unanimement sur l'irrigation. Les années 1995 à 1997 plus conformes ont quelque peu rasséréné les esprits.

2. Chiffres fournis par L. Davy (1990), p. 99.

3. E. About (1907), p. 96. Le célèbre auteur du Roi des montagnes fut l'un des plus lucides observateurs de la Grèce au milieu du XIX ${ }^{\mathrm{e}}$ siècle.

4. Formation rocheuse constituée d'une alternance de lits calcaires perméables et de lits marneux, souvent gréseux imperméables, présentant un aspect de mille feuille.

5. La densité de population est partout inférieure à 19 hab. $/ \mathrm{km}^{2}$.

6. J. M. Bernabé Y Maestre (1989).

7. Terme dépréciatif d'origine ottomane désignant les sujets non musulmans de l'Empire. Ici, par extension dans le vocabulaire populaire, il qualifie péjorativement les paysans sédentaires des plaines par opposition aux pasteurs nomades ou seminomades.

8. C'est le cas des communes bordières au sud du lac Trichonída, tandis que les Kravarites doivent s'expatrier, à l'instar des habitants de Livadáki (anciennement Avórani) s'en allant fonder Néa Avórani près d'Agrínio et conservant le nom originel de leur village.

9. Pour couvrir les besoins de la Grèce, pauvre en ressources énergétiques, et pour exporter du courant électrique contre des devises.

10. Pour expliquer ces soixante ans d'écart entre les premières études et le début de la réalisation du barrage de Kremastá, il suffit de se rappeler l'histoire tourmentée de la Grèce entre la Grande Catastrophe d'Asie Mineure et la guerre civile. La région a été en outre un lieu d'affrontement entre les résistants et les troupes d'occupation durant la guerre et entre les partisans communistes et les troupes royales pendant la guerre civile. 
11. Il s'agit des villages de Tríklinio, d'Alevráda, sur la rive droite de l'Achelóos, et d'Agalianós, de Psilóvrachos et de Charavghí, sur la rive gauche de la Mégdovas.

12. E. D. Karaghéorgou (1966) : « Il est urgent de trouver un nouveau lieu sûr avant que les hommes n'éprouvent le besoin de quitter le village [de Tríklinio] et de s'expatrier dans les centres urbains ou à l'étranger.»

13. Ainsi, par décision ministérielle du 18 juin 1969, 185 habitants d'Alevráda et 148 de Petróna sont déplacés au hameau de Triandéíka, commune d'Eleófyto, qui voit sa population doubler entre 1961 et 1971.

14. Soit environ 9200 personnes issues de 19 communes.

15. L'évolution moyenne pour les communes de montagne valtinotes ressort à - $31 \%$. 16. Organisme Général d'Amélioration Foncière, service public chargé de distribuer l'eau d'irrigation, de promouvoir et d'entretenir les canaux et de susciter les travaux de bonification. Il regroupe les organismes syndicaux locaux présents à l'échelon communal, les TOEV.

17. À titre d'exemple le tarif forfaitaire était en 1992 de 3000 drachmes par strémma pour l'année et moitié prix si le champ était équipé d'un système d'arrosage au goutte à goutte.

18. La compagie nationale grecque d'électricité.

19. Plan Intégré Méditerranéen.

20. Le strémma est l'unité de surface couramment utilisée en agriculture, correspondant à 0,1 ha (pluriel : strémmata).

21. La DEYAA est l'entreprise publique des eaux et d'épuration d'Agrínio.

22. L'annuaire statistique pour l'année 1988 publié en 1990, ne mentionne pour les rejets dans les fleuves et les lacs, que quelques éléments mesurés sur l'Achelóos à

Kastráki, soit à l'amont des principales zones de rejets agricoles et urbains. Rien sur le lac Trichonída! Dans ces conditions, la qualité de l'eau apparaît relativement satisfaisante (p. 54).

23. Par endroits dans K. Arvanítis et A. Gatzélia (1990).

24. Je construis cette expression à partir du mot paréa désignant en grec le groupe de copains ou d'amis, mais dont la réalité est intraduisible en français. Elle ne correspond pas exactement à une amicale qui suppose un centre d'intérêt a priori.

25. Par exemple procès au civil d'un TOEV d'une commune proche d'un lac artificiel contre la DEI pour obtenir un meilleur entretien des canaux d'irrigation contractuellement à la charge de la compagnie d'électricité gestionnaire du barrage. Dans d'autres cas, des TOEV intentent des actions en récupération des sommes dues par des adhérents mauvais payeurs.

26. Voir M. Drain (dir.) (1996).

27. Avec le retour d'une pluviométrie plus abondante en 1994 et 1995, ce projet serait abandonné.

28. Ainsi, lors du débat parlementaire sur le vote du budget 1997, retransmis par la télévision en décembre 1996, un député thessalien de la majorité s'est-il félicité de ces travaux. Ce que ses homologues des nomes occidentaux se sont bien gardés de faire! 29. Cité par D. Loukópoulos (1983), p. 13 et que l'on peut encore entendre. 


\section{RÉSUMÉS}

L'Étolie-Acarnanie, véritable château d'eau de la Grèce dispose de ressources abondantes par ses cours d'eau torrentiels et ses lacs, lesquels couvrent un quart de la surface lacustre hellénique. Sont venus s'y ajouter, depuis une trentaine d'années, des lacs de retenue parmi les plus imposants du pays.

L'exploitation traditionnelle de l'eau était le fait de techniques individuelles, rudimentaires et ingénieuses, fruit essentiellement des efforts individuels ou de communautés villageoises. Elles consistent dans le captage de multiples sources en Étolie montagnarde et par les loútses en AcarnanieLes plaines paludéennes, progressivement drainées à partir du siècle dernier et les lacs ont permis l'extension de la culture spéculative du tabac. Plus récemment, les forages individuels ont complété le dispositif. La vision du monde engendrée par le système agro-sylvo-pastoral s'est maintenue en plaine dans un cadre spécifiquement agricole et spéculatif. Le cœur en est constitué par la maîtrise de l'apport hydrique, ainsi que par la prééminence de l'effort individuel reposant sur une conception viscérale de la liberté et une méfiance séculaire envers l'État.

L'État hellénique a été le maître d'ouvrage, depuis le début des années soixante, de barrages gigantesques sur l'Achelóos notamment pour l'électrification du pays, puis en détournant une partie du flot vers la Thessalie et enfin sur l'Évinos pour pourvoir l'Attique en eau. Les besoins locaux n'ont pas été pris en compte. Le mécontentement, dépassant de beaucoup les protestations de quelques organisations écologistes allemandes, a pris des formes diverses. Les manifestations sont négatives (procès, contestation) ou affirmatives (repli sur un système de débrouillardise individuelle). Elles intègrent des revendications économiques, écologiques essentiellement patrimoniales - et une affirmation identitaire contre Athènes et la Thessalie.

Aetolia-Acarnania, real Greece's water tower thanks to its position on the occidental front of the country, has abundant resources with its torrential waterways and its lakes, which are covering one quarter of the hellenic lacustral surface. Since about thirty years, many dam lakes amongst the biggest of the country had been added.

The traditional tapping of water was made of individual, rudimentary and clever technologies essentially fruit of individual or community efforts as part of the village. They consist in doing harnessing of multiple sources in mountainous Aetolia or in loútses in Acarnania. Marshy plains, progressively drained since the last century have allowed the extension of the speculative tobacco cultivation. More recently, individual drillings completed this apparatus. The world vision produced by the agricultural-forester-pastoral system is maintained in plains in spite of agricultural and speculative activities. In the depth of this world vision is the mastership of bringing in water, as well as the pre-eminence of individual effort based on a visceral conception of freedom and a century-old mistrust towards State.

Hellenic State was the work master, since the beginning of the " 60 , for building giant dams on the Achelóos, more especially to electrify the country, afterwards to turn away a major part of the flow towards Thessaly plain, and at last on the Évinos to bring water to Attica. Local needs have been neglected. Dissatisfaction, overstepping by far protests from some german ecologist organizations, took diverse forms. Manifestations are negative (action, dispute) or affirmative (turning up an individual resourceful system). They integrate into themselves economic, ecologic - essentially patrimonial - claims and affirmation of its own identity against Athens and Thessaly. 
INDEX

Index géographique : Grèce

Mots-clés : Eau

AUTEUR

MICHEL BOUILLET

Professeur agrégé 